\title{
Efeitos da Histerectomia Total Abdominal sobre o Fluxo Sangüíneo Ovariano
}

\author{
Effect of Total Abdominal Hysterectomy on Ovarian Blood Supply
}

\author{
Eliana Aguiar Petri Nahás ${ }^{1}$, Anaglória Pontes ${ }^{1}$, Paulo Traiman ${ }^{1}$, Jorge Nahás Neto ${ }^{1}$, \\ Laurival A. De Luca ${ }^{1}$, Vera Therezinha Medeiros Borges ${ }^{2}$ \\ Lídia Raquel de Carvalho ${ }^{3}$
}

\begin{abstract}
RESUM0
Objetivo: avaliar os efeitos da histerectomia total abdominal (HTA) sobre o fluxo sangüineo ovariano, em mulheres no menacme, por meio da dopplervelocimetria e ultra-sonografia transvaginal.

Métodos: estudo prospectivo no qual foram incluidas 61 mulheres, com idade igual ou inferior a 40 anos. As pacientes foram divididas em dois grupos: G1, com 31 pacientes submetidas à HTA, e G2, com 30 mulheres normais não submetidas à cirurgia. Somente foram incluidas pacientes eumenorréicas, ovulatórias, não-obesas ou fumantes, sem cirurgias ou doenças ovarianas prévias. Avaliou-se o fluxo sangüíneo das artérias ovarianas, inicialmente e aos 6 e 12 meses, pelo índice de pulsatilidade (IP) na dopplervelocimetria, e o volume ovariano pela ultra-sonografia transvaginal (US). Para análise estatística empregou-se teste t pareado, análise de perfil, teste de Friedman e teste de Mann-Whitney.

Resultados: na comparação estatística inicial os grupos foram homogêneos quanto às características epidemiológicas e quanto aos demais parâmetros avaliados neste estudo. Nas pacientes submetidas à histerectomia, observaram-se aos 6 e 12 meses aumento do volume ovariano ao US e diminuição do IP avaliado pela dopplervelocimetria ( $p<0,05)$, quando confrontadas ao controle. Aos 12 meses, em 8 das 31 pacientes pós-HTA (25,5\%) verificouse ocorrência de cistos ovarianos de aspecto benigno. No grupo controle não houve alteração de nenhum desses parâmetros.

Conclusão: a redução do IP na dopplervelocimetria das artérias ovarianas sugere aumento do fluxo sangüíneo ovariano pós-histerectomia total abdominal em mulheres no menacme.
\end{abstract}

PALAVRAS-CHAVE: Histerectomia total abdominal. Dopplervelocimetria. Cistos de ovários.

\section{Introdução}

A preservação ou remoção dos ovários é decisão controversa para o ginecologista que realiza a histerectomia total abdominal (HTA) ${ }^{1-3}$. A média

\footnotetext{
${ }^{1}$ Disciplina de Ginecologia

${ }^{2}$ Setor de Ultra-sonografia do Departamento de Ginecologia e Obstetrícia

${ }^{3}$ Departamento de Bioestatística

Faculdade de Medicina de Botucatu - UNESP

Correspondência:

Eliana Aguiar Petri Nahás

Departamento de Ginecologia e Obstetrícia

Faculdade de Medicina de Botucatu - UNESP

18618-000 - Rubião Júnior - Botucatu - SP

Fone: (14) 6802-6227 - Fax: (14) 6822-1933

e-mail: epetri@fmb.unesp.br
}

de idade das pacientes submetidas a esta cirurgia é de 45 anos, com $68 \%$ dos casos entre 35 e 50 $\operatorname{anos}^{3}$. Para Laughlin et al. ${ }^{4}$, a ooforectomia profilática quando da HTA priva grande número de mulheres dos efeitos benéficos dos esteróides sexuais endógenos e as conseqüências da redução dos androgênios ovarianos após a menopausa são pouco conhecidas. Em contrapartida, observase redução no risco de câncer de ovário em mulheres submetidas à histerectomia. Este efeito talvez se deva às alterações do fluxo sangüíneo ovariano, pela diminuição da exposição a carcinógenos vaginais e/ou pela detecção de doença ovariana oculta no ato cirúrgico ${ }^{5,6}$.

O trauma cirúrgico da HTA comprometeria a irrigação sangüínea ovariana, com subseqüen- 
te alteração da função dos ovários ${ }^{7}$. O suprimento sangüineo de tubas e ovários deriva-se das artérias uterinas e ovarianas. A artéria ovariana, originária da aorta, está presente no ligamento infundíbulo-pélvico e divide-se em três ramos. O ramo lateral do ovário anastomosa-se com um ramo da artéria uterina, o ovariano medial, situada ao longo do ligamento útero-ovariano no mesovário, formando a arcada ovariana ${ }^{8}$. Janson e Janson ${ }^{9}$ avaliaram o fluxo ovariano, imediatamente após a HTA, utilizando-se do radioisótopo $\mathrm{Xe}^{133}$. Observaram redução na taxa de clearance ovariano de 50 a $90 \%$, o que sugere diminuição aguda da perfusão ovariana decorrente da ligação dos ramos anexiais das artérias uterinas. Este procedimento elevaria a pressão intra-ovariana, com lesão da microcirculação dos ovários e conseqüentemente insuficiência ovariana ${ }^{10,11}$.

Durante a última década, se expandiu o emprego da ultra-sonografia transvaginal com dopplervelocimetria na avaliação da hemodinâmica ovariana durante o ciclo menstrual. As variações no padrão da velocidade de fluxo nas artérias pélvicas, detectadas pela dopplervelocimetria, são decorrentes de fatores hormonais e angiogênicos. $\mathrm{Na}$ ovulação, a resistência dos vasos ovarianos diminui, sob a influência dos esteróides sexuais, havendo aumento do fluxo sangüineo para os ová$\operatorname{rios}^{12-15}$. Kurjak e Kupesic ${ }^{15}$ mediram a velocidade de fluxo ovariano em mulheres no menacme e na menopausa. Observaram que os sinais de fluxo diastólico arterial ovariano, ao Doppler, reduzemse progressivamente com a idade, tornando-se ausentes cinco anos após a menopausa.

A medida da velocidade de fluxo baseia-se no efeito Doppler descrito por Christian Doppler (18031853). São utilizados vários indices para análise das variações da velocidade de fluxo, como o índice de pulsatilidade (IP) e o índice de resistência, que proporcionam resultados confiáveis e comparáveis a respeito dos eventos circulatórios ${ }^{16}$. Cada vaso sangüíneo da pelve tem padrão particular de onda. A artéria ovariana apresenta fluxo de baixa velocidade, com resistência variável, de acordo com a fase do ciclo $^{12,16}$. O IP é provavelmente o de maior acurácia na detecção das alterações de pulsatilidade e resistência periférica, sendo esta inversamente proporcional ao fluxo ${ }^{17}$. Dogan et al. ${ }^{18}$ utili- $^{-}$ zando-se da dopplervelocimetria, avaliaram a perfusão dos ovários antes e após 6 meses de histerectomia total abdominal, em 43 mulheres com idade inferior a 45 anos. Não demonstraram nenhuma diferença significativa no índice de resistência das artérias ovarianas entre o pré e o pósoperatório ou quando comparado ao grupo controle. Esses autores concluem que o fluxo ovariano não se altera no prazo de seis meses após HTA.
Até o presente momento pouco se conhece sobre o padrão da velocidade de fluxo nas artérias ovarianas pós-HTA. Assim, este estudo teve como objetivo avaliar a fluxo sangüíneo ovariano pela dopplervelocimetria, em mulheres no menacme, submetidas a histerectomia total abdominal.

\section{Pacientes e Métodos}

Foi conduzido estudo prospectivo, tipo casocontrole e longitudinal, no qual foram incluídas 61 pacientes, com idade inferior ou igual a 40 anos, que foram divididas em dois grupos: grupo 1 (G1), composto por 31 pacientes submetidas à histerectomia total abdominal por doenças benignas do útero, e grupo 2 (G2), no qual foram incluídas 30 mulheres normais (controle). Somente foram aceitas no estudo mulheres eumenorréicas, com ciclos ovulatórios (valores de progesterona superior a $5 \mathrm{ng} / \mathrm{mL}$, na fase lútea), sem história de cirurgia prévia, laqueadura tubária ou doença ovariana. Nenhuma mulher usava medicação que influenciasse os resultados há pelo menos seis meses. Foram excluídas as pacientes fumantes e/ou obesas. Todas as pacientes assinaram o consentimento esclarecido. O projeto de pesquisa recebeu parecer favorável do Comitê de Ética em Pesquisa da Faculdade de Medicina de Botucatu.

Preliminarmente, as pacientes foram submetidas a anamnese e exame físico geral e ginecológico. Os seguintes dados foram coletados: idade, menarca, caracterização do ciclo menstrual, paridade, peso e estatura. Foi colhida colpocitologia oncológica e realizada a ultra-sonografia transvaginal. No grupo de pacientes submetidas à cirurgia, as queixas pré-operatórias foram: hipermenorragia $(77,4 \%)$, dismenorréia secundária $(54,8 \%)$, dor pélvica $(19,3 \%)$, polimenorréia $(12,9 \%)$ e aumento do volume abdominal $(9,6 \%)$. A maioria das pacientes relatavam mais que um sintoma. O tempo mé-

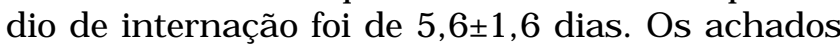
histopatológicos mostraram 21 casos de leiomiomas $(67,7 \%), 5$ de adenomiose $(16,1 \%), 3$ de hipertrofia uterina difusa $(9,6 \%)$ e em 2 casos úteros normais $(6,4 \%)$. O tempo de seguimento para ambos os grupos foi de 12 meses.

Realizou-se a ultra-sonografia transvaginal para medição do volume dos ovários e a dopplervelocimetria para avaliação da forma de onda da velocidade do fluxo ovariano medido pelo IP, basal e aos 6 e 12 meses. Todos os exames foram realizados na fase folicular do ciclo menstrual, pelo mesmo examinador, no período da tarde. Para estabelecimento da fase folicular, nas pacientes histerectomizadas, considerou-se a ausência de 
folículo dominante e do corpo lúteo. O tempo médio de duração do exame foi de 20 minutos. Utilizou-se o aparelho Sonochrome ${ }^{\circledR}\left(\mathrm{GE}^{\circledR}\right.$, USA) com transdutor endovaginal de $7,5 \mathrm{MHz}$, para imagem morfológica de útero e ovários, e Doppler colorido. No exame basal, foram medidos o diâmetro transverso (T), o ântero-posterior (AP) e o longitudinal (L) do útero e ovários, obtendo-se o volume (V) estimado segundo a fórmula $\mathrm{V}\left(\mathrm{cm}^{3}\right)=\mathrm{T}(\mathrm{cm}) \times \mathrm{AP}$ $(\mathrm{cm}) \times \mathrm{L}(\mathrm{cm}) \times 0,52$. Observou-se o fluxo sangüíneo das artérias ovarianas direita e esquerda, no infundíbulo pélvico, pelo mapeamento colorido, obtendo-se o típico espectro de velocidade deste vaso. A impedância do fluxo sangüíneo foi expressa pelo IP. Este foi calculado, eletronicamente, de acordo com a fórmula IP $=\mathrm{S}-\mathrm{D} /$ média, onde S é o pico de velocidade sistólica, D é a velocidade diastólica mínima, sobre a média da velocidade de fluxo. Para o cálculo apropriado, foram registradas pelo menos cinco ondas da velocidade de fluxo similares e de qualidade satisfatória.

Para comparação das características clínicas e ultra-sonográficas iniciais entre os grupos, realizou-se o teste $t$ pareado. Para a variável IP, que apresentou distribuição normal, utilizou-se a análise de perfil, seguida do método de comparações múltiplas de Tukey, com os valores expressos pela média e desvio-padrão. Para as medidas do volume ovariano, que demonstrou ter distribuição não normal, foram aplicados o teste de Friedman e o teste de Mann-Whitney, com os resultados em mediana e valores mínimo e máximo. O nível de significância escolhido foi de 5\% e os dados calculados com o auxílio do Software SAS Versão 6.3.

\section{Resultados}

As características clínicas e ultra-sonográficas iniciais das pacientes submetidas à HTA e do grupo controle foram submetidas a comparação estatística, e estão representadas na Tabela 1 . Verificou-se que os grupos foram homogêneos para as seguintes variáveis: idade, menarca, paridade, intervalo do ciclo menstrual, peso, altura e índice de massa corpórea (IMC). Em relação às medidas ultra-sonográficas, apenas o volume uterino diferiu entre os grupos. As pacientes do grupo de histerectomia apresentavam no pré-operatório valores significativamente maiores quando comparados ao grupo controle (Tabela 1).

$\mathrm{Na}$ análise do IP, pela dopplervelocimetria, nas pacientes submetidas a histerectomia, encontrou-se redução significativa nos valores do IP, aos 6 e 12 meses, em ambos os ovários, quando com- parado ao grupo controle ( $\mathrm{p}<0,05)$ (Figura 1). Aos 6 meses, não foi possivel localizar os vasos ovarianos para mensuração do IP em 6,4\% (4/62) dos exames realizados no grupo de pacientes histerectomizadas e em $6,7 \%$ (4/60) do controle, e aos 12 meses, em 3,2\% (2/62) e 5,0\% (3/60), respectivamente.

Tabela 1 - Comparação das características clínicas e ultra-sonográficas iniciais entre as pacientes no menacme submetidas à histerectomia total abdominal (HTA) $(n=31)$ e 0 grupo controle $(n=30)$ (valores médios \pm desvio-padrão)

\begin{tabular}{lrr}
\hline Variáveis & \multicolumn{1}{c}{ HTA } & \multicolumn{1}{c}{$\begin{array}{c}\text { Controle } \\
\text { (G1) }\end{array}$} \\
\hline Idade (anos) & $37,6 \pm 2,6$ & $36,7 \pm 2,9$ \\
Idade da menarca (anos) & $12,8 \pm 1,6$ & $12,7 \pm 1,5$ \\
Paridade (nº de filhos) & $2,2 \pm 1,4$ & $1,8 \pm 1,8$ \\
Intervalo menstrual (dias) & $28,4 \pm 1,9$ & $28,4 \pm 2,1$ \\
IMC (kg/m²) & $25,6 \pm 3,2$ & $24,8 \pm 2,8$ \\
Ovário direito (cm ${ }^{3}$ ) & $6,0 \pm 2,4$ & $7,0 \pm 2,1$ \\
Ovário esquerdo (cm $\left.{ }^{3}\right)$ & $6,4 \pm 2,9$ & $6,8 \pm 2,5$ \\
Doppler direito (IP) & $2,6 \pm 0,6$ & $2,9 \pm 0,8$ \\
Doppler esquerdo (IP) & $2,9 \pm 0,6$ & $3,0 \pm 0,8$ \\
Útero (cm ${ }^{3}$ ) & $309,0 \pm 243,6$ & $92,2 \pm 5,9 *$
\end{tabular}

* $\mathrm{p}<0,05$ (test $t$ pareado)

IMC = Índice de Massa Corpórea

IP = Índice de Pulsatilidade

IPD
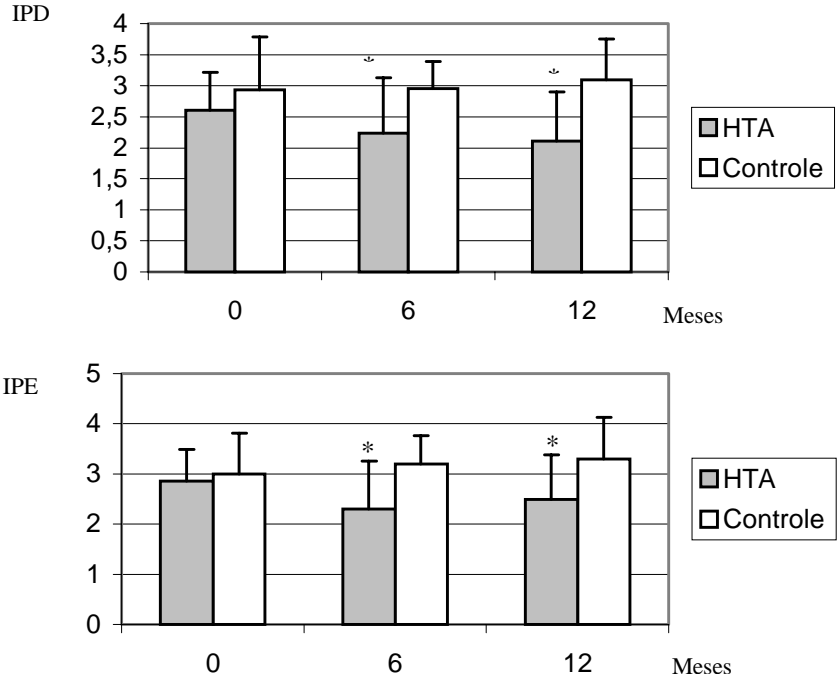

Figura 1 - Comparação do índice de pulsatilidade (IP) das artérias ovarianas direita (D) e esquerda $(E)$, entre as pacientes no menacme submetidas à histerectomia total abdominal (HTA) ( $n=31)$ e 0 grupo controle $(n=30)$ ( valores médios \pm desvio padrão). ${ }^{*} p<0,05$ (análise de perfil)

Nas pacientes submetidas à HTA, a ultrasonografia mostrou aumento significante do volume mediano dos ovários direito e esquerdo, aos 6 e 12 meses, quando comparado ao controle $(\mathrm{p}<0,05)$ 
(Figura 2). Aos 6 meses, 41,9\% (13/31) das pacientes submetidas à cirurgia apresentavam cistos funcionais ovarianos, com volume de $27 \mathrm{~cm}^{3}$ a 324 $\mathrm{cm}^{3}$, mais freqüentes à esquerda (9/13). Todavia, aos 12 meses, em 10 desses 13 casos $(76,9 \%)$ não se evidenciavam mais os referidos cistos. Por outro lado, nesse período, 5 novos casos surgiram, com volume de $36,5 \mathrm{~cm}^{3}$ a $82 \mathrm{~cm}^{3}$. Portanto, aos 12 meses, em 25,5\% (8/31), ainda se encontravam cistos ovarianos, de aspecto benigno. Os ovários do grupo controle mantiveram-se com dimensões normais durante o seguimento (Figura 2).
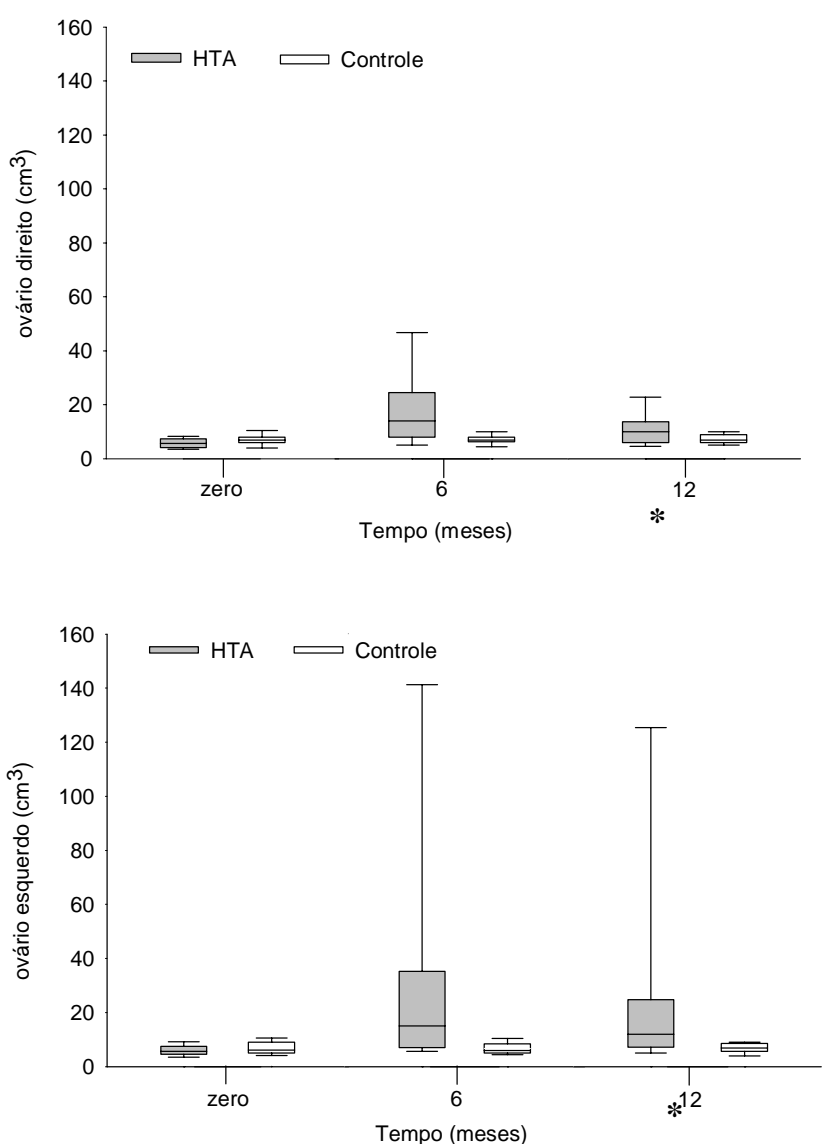

Figura 2 - Comparação entre os valores do volume ovariano $\left(\mathrm{cm}^{3}\right)$ direito e esquerdo, entre as pacientes no menacme submetidas à histerectomia total abdominal $(\mathrm{HTA})(\mathrm{n}=31)$ e 0 grupo controle $(n=30)$ (valores expressos em mediana e percentis 10, 25, 75 e 90). * $p<0,05$ (teste de Mann-Whitney)

Uma paciente que aos 6 meses apresentava volume ovariano de $324 \mathrm{~cm}^{3}$, aos 12 meses relatava dor na fossa ilíaca esquerda, com tumoração ao exame ginecológico. A ultra-sonografia mostrou imagem cística, de $421 \mathrm{~cm}^{3}$, na região anexial esquerda. Os marcadores tumorais CA-125, proteína $\mathrm{C}$ reativa e antígeno carcinoembriogênico (CEA) foram negativos. Realizou-se laparotomia exploradora. O achado cirúrgico revelou cisto de retenção à esquerda, e o exame histopatológico exibiu a tuba com fibrose peritubária e ovário esquerdo com folículos císticos, corpo lúteo e albicans.

\section{Discussão}

Alguns estudos indicam que a incidência de falência ovariana em mulheres submetidas a HTA é maior que a observada na população em geral ${ }^{7,11}$, ao passo que outros não observam alteração da função ovariana ${ }^{10,19}$. Uma hipótese para o aumento na incidência de falência ovariana pós-histerectomia é o estiramento dos vasos no infundíbulo pélvico, seguido de trombose e redução do fluxo sangüíneo ovariano ${ }^{10,11,19}$. Para investigar essa hipótese, avaliamos neste estudo a circulação ovariana, antes e após a cirurgia, pela dopplervelocimetria das artérias ovarianas, na fase folicular do ciclo menstrual. Neste período o fluxo é menor e similar em ambos os ovários, ao passo que imediatamente antes da ovulação e na fase lútea inicial, há maior perfusão, principalmente no ovário dominante, com redução nos valores do IP e da impedância vascular ${ }^{12,13}$.

Os valores iniciais do IP foram semelhantes entre os grupos. Todavia, nas pacientes submetidas à histerectomia, ocorreu redução significativa nos valores médios do IP nas artérias ovarianas direita e esquerda, aos 6 e 12 meses, sugerindo aumento na perfusão sangüínea dos ovários, quando confrontadas ao controle. Esse dado contrapõe-se à hipótese de redução de fluxo sangüíneo ovariano após HTA. Até o momento, a literatura carece de estudos referentes à perfusão ovariana pós-histerectomia, avaliada pela dopplervelocimetria. No único estudo, os autores não demonstraram alterações significativas no fluxo ovariano, pelo índice de resistência, 6 meses após a cirurgia $^{18}$.

O volume ovariano inicial nas pacientes deste estudo foi em média de $6,5 \mathrm{~cm}^{3}$, sendo este resultado concordante com a literatura ${ }^{20}$. Pavlik et al. ${ }^{20}$, estudando o volume dos ovários, pela ultrasonografia transvaginal, em 58.673 mulheres com idade entre 25 a 91 anos, estabeleceram o limite superior de $20 \mathrm{~cm}^{3}$ no menacme e de $10 \mathrm{~cm}^{3}$ na menopausa. O volume médio foi de $6,1 \mathrm{~cm}^{3}$ nas pacientes entre 30 e 39 anos. Esses autores notaram redução significativa do volume ovariano a cada década de vida. Nesta investigação, após a HTA, observou-se aumento significativo do volume mediano dos ovários, aos 6 e 12 meses. A elevação do fluxo, demonstrado pela redução do IP ao Doppler, possivelmente seja reflexo do maior tamanho dos ovários após a cirurgia. No grupo con- 
trole os ovários não mostraram alterações ao longo do seguimento.

No seguimento pós-cirúrgico das pacientes deste estudo, ao final de 12 meses, 25,5\% (8/31) apresentavam cistos funcionais na ultra-sonografia. Apenas uma paciente $(3,2 \%)$ submeteu-se a laparotomia exploradora, que revelou aderências e cisto de inclusão, e não aumento do volume ovariano. A síndrome do ovário residual, que inclui massa pélvica persistente, dor e/ou dispareunia, é relatada em mulheres submetidas a HTA, com incidência variável de 1 a $3 \%$. Reflete possível disfunção ovariana secundária a aderências e periooforites, que interferem no desenvolvimento folicular ${ }^{21}$. O diagnóstico de certeza é feito somente com a resolução dos sintomas após a ooforectomia ${ }^{1}$. O achado histopatológico mais comum são cistos funcionais, em mais de $50 \%$ dos casos, com fisiopatologia desconhecida ${ }^{1,22}$. A formação desses cistos é freqüente dentro do primeiro ano pós-cirurgia, com resolução espontânea na maioria dos casos $^{19}$, como demonstrado nesta investigação. Mas, 5\% a $8 \%$ das pacientes, após HTA, necessitam de cirurgias subseqüentes por patologias benignas ovarianas ${ }^{3}$. Menon et al. ${ }^{23}$ evidenciaram cistos ovarianos na ultra-sonografia em 30\% (18) 60) dos casos, mais freqüentes dentro do primeiro ano de cirurgia, com ooforectomia em 5\% destes casos. Zalel et al. ${ }^{24}$, em estudo prospectivo, acompanharam com exames ultra-sonográficos periódicos durante 4 anos, 73 mulheres com média de idade de 44,5 anos, que haviam sido submetidas a histerectomia. Em 37 casos (50,7\%) encontraram cistos ovarianos. Destas, quatro $(5,5 \%)$ submeteram-se a laparotomia exploradora. Os achados histopatológicos foram dois casos de cisto paraovariano, um cistoadenoma e uma hidrossalpinge com aderências periovarianas. Pete e Bõsze ${ }^{25}$ observaram cistos em 9,2\% (6/65) das pacientes submetidas à HTA, e 4,6\% (3/65) foram submetidas a nova cirurgia, no período de 3 anos.

Baseados nos resultados do presente estudo, constatou-se redução no IP, refletindo elevação no fluxo sangüíneo nas artérias ovarianas pós histerectomia. Esse dado contrapõe-se à hipótese de déficit de fluxo sangüíneo como fator causal de possivel insuficiência ovariana pós-histerectomia total abdominal em mulheres no menacme.

\section{ABSTRACT}

Purpose: to evaluate the effect of total abdominal hysterectomy on ovarian blood supply in women in reproductive age, using transvaginal color Doppler.
Methods: a prospective study was carried out on 61 40-yearold or younger women. They were divided into two groups: G1, 31 patients submitted to hysterectomy, and G2, 30 normal women. Criteria of inclusion: normal ovarian function at baseline, normal body weight, without expasure to tobacco, no history of laparotomy or ovarian pathology. The pulsatility index (PI) of ovarian arteries determined by Doppler and the transvaginal ultrasonographic measurement of the ovarian volume were performed at three moments: baseline, 6 and 12 months.

Results: at baseline the groups were homogeneous. In the hysterectomized group, with 6 and 12 months, increase of the volume of the ovaries and decrease of $P I(p<0,05)$ were observed. At the end of the study, 25.5\% (8/31) of the hysterectomized patients presented benign ovarian cysts. In the control group no alteration of the parameters was observed.

Conclusion: The decrease of PI by Doppler of ovarian arteries suggests increased ovarian blood supply after total abdominal hysterectomy in women in reproductive age.

KEY WORDS: Total abdominal hysterectomy. Doppler ultrasonography. Ovary.

\section{Referências}

1. Dekel A, Efrat Z, Orvieto R, et al. The residual ovary syndrome: a 20-year experience. Eur J Obstet Gynecol Reprod Biol 1996; 68:159-64.

2. Fong YF, Lim FK, Arulkumaran S. Prophylactic oophorectomy: a continuing controversy. Obstet Gynecol Surv 1998; 53:493-9.

3. Reich H. Issues surrounding surgical menopause. J Reprod Med 2001; 46:297-306.

4. Laughlin GA, Barrtt-Connor E, Silverstein D, von Mühlen D. Hysterectomy, oophorectomy, and endogenous sex hormone levels in older women: The Rancho Bernardo Study. J Clin Endocrinol Metab 2000; 85:645-51.

5. Parazzini F, Negri E, Vecchia C, Luchini L, Mazzopane R. Hysterectomy, oophorectomy, and subsequent ovarian cancer risk. Obstet Gynecol 1993; 81:363-6.

6. Beard CM, Hartmann LC, Atkinson EJ, et al. The epidemiology of ovarian cancer: a population-based study in Olmsted County, Minnesota, 1935-1991. Ann Epidemiol 2000; 10:14-23.

7. Chalmers C. Does hysterectomy in premenopausal woman affect ovarian function? Med Hypotheses 1996; 46:573-5.

8. Borell U, Fernström I. The adnexal branches of the uterine artery. Acta Radiol 1953; 40:561-82.

9. Janson PO, Janson I. The acute effect of hysterectomy on ovarian blood flow. Am J Obstet Gynecol 1977; 127:349-52. 
10.Souza AZ, Fonseca AM, Izzo VM, Clauzet RM, Salvatore CA. Ovarian histology and function after total abdominal hysterectomy. Obstet Gynecol 1986; 68:847-9.

11.Siddle N, Sarrel P, Whitehead M. The effect of hysterectomy on the age at ovarian failure: identification of a subgroup of women with premature loss of ovarian function and literature review. Fertil Steril 1987; 47:94-100.

12.Hata K, Hata T, Senoh D, et al. Change in ovarian arterial compliance during the human menstrual cycle assessed by Doppler ultrasound. Br J Obstet Gynaecol 1990; 97:163-6.

13.Tinkanen H, Kujansuu E, Laippala P. The association between hormone levels and vascular resistance in uterine and ovarian arteries in spontaneous menstrual cycles - a Doppler ultrasound study. Acta Obstet Gynecol Scand 1995;74:297-301.

14.Tan SL, Zaidi J, Campbell S, Doyle P, Collins W. Blood flow changes in the ovarian and uterine arteries during the normal menstrual cycle. Am J Obstet Gynecol 1996; 175:625-31.

15.Kurjac A, Kupesic S. Ovarian senescence and its significance on uterine and ovarian perfusion. Fertil Steril 1995; 64:532-7.

16.Serafini P, Batzofin J. Transvaginal color Doppler ultrasonography in reproductive gynecology. Int $\mathrm{J}$ Fertil 1994; 39:253-61.
17.Prömpeler HJ, Madjar H, Sauerbrei W. Classification of adnexal tumors by transvaginal color Doppler. Gynecol Oncol 1996; 61:354-63.

18.Dogan MM, Basaran Z, Ekici E, et al. Effect of hysterectomy on ovarian blood supply and function. J Obstet Gynaecol 1998; 18:263-6.

19.Bhattacharya S, Mollison J, Pinion S, et al. A comparison of bladder and ovarian function two years following hysterectomy or endometrial ablation. Br J Obstet Gynaecol 1996; 103:898-903.

20.Pavlik EJ, DePriest PD, Gallion HH, et al. Ovarian volume related to age. Gynecol Oncol 2000; $77: 410-2$

21.Grogan RH. Reappraisal of residual ovaries. Curr Opin Obstet Gynecol 1967; 97:124-9.

22.Richlin SS, Rock JA. Ovarian remnant syndrome. Gynaecol Endoscopy 2001; 10:111-7.

23. Menon RK, Okonofua FE, Agnew JE, et al. Endocrine and metabolic effects of simple hysterectomy. Int $\mathrm{J}$ Gynaecol Obstet 1987; 25:459-63.

24.Zalel Y, Lurie S, Beyth Y, Goldberger S, Tepper R. Is it necessary to perform a prophylactic oophorectomy during hysterectomy? Eur J Obstet Gynecol Reprod Biol 1997; 73:67-70.

25.Pete I, Bõsze P. The fate of the retained ovaries following radical hysterectomy. Eur J Gynaecol Oncol 1998; 19:22-4.

\section{Congresso Paulista de Obstetńcia e Ginecologia}

\section{5 a 18 de agosto de 2002}

\section{São Paulo - SP}

\section{Informações: SOGESP \\ (11) 5182-7867 - 5182-0573}

